\title{
Driver's Attitudes toward the Safety of In-vehicle Navigation Systems
}

\author{
Andrew Varden, Jonathan Haber and Blair Nonnecke \\ Department of Computing \& Information Science, University of Guelph
}

Copyright @ 2008 SAE International

\begin{abstract}
There is anecdotal evidence of drivers blindly following in-vehicle navigation system (IVNS) commands. IVNSs have shown to be distracting and mishaps with the device have entered popular culture as a source of comedy. Manufactures have reacted by warning drivers of the dangers involved in operating the devices, and in some cases prevent address input while moving. While IVNSs are increasingly being used, do drivers perceive their use as distracting, potentially misleading and thus dangerous?
\end{abstract}

We conducted an online survey of over 200 drivers to determine their attitudes toward safety while using these devices. This was followed by a series of interviews with an additional 20 drivers to provide more in-depth results. Drivers reported that distraction is not a big issue for them when using an IVNS, with only $8 \%$ reporting that the device was too distracting at times. Over $90 \%$ of respondents believe IVNSs do not have a harmful or potentially injurious effect and they are not wary of the device. They also placed more trust in directions from IVNSs than from people. There is a discrepancy between drives' attitudes towards safety and potential dangers of using an IVNS. Drivers may be unaware of how distraction affects their driving. Some did not feel using an IVNS was dangerous at all because they are ultimately responsible any incidents while driving.

\section{INTRODUCTION}

IVNS combine information from GPS satellites and electronic maps to provide turn-by-turn directions. Both sources of information are fairly reliable, but not perfect. The systems seem to perform well enough that in recent years the popularity of IVNS has increased significantly. The increased use of IVNSs has led to some concerns about safety. There are concerns about drivers operating the devices while driving that has resulted in some IVNS manufacturers locking out certain functions when the car is moving. There is also concern about drivers becoming distracted by the IVNS while driving, and drivers following IVNS directions that are incorrect.
The concern that using a cell phone while driving is unsafe resulted in laws that ban the practice in some jurisdictions [1]. There are safety concerns when using an IVNS, including the potential for distraction. Even though an IVNS is a tool designed to help drivers there is still the possibility that they will be misused.

The following is an excerpt from a product safety information sheet produced by an IVNS manufacturer:

When navigating, carefully compare information displayed on the unit to all navigation sources, including information from street signs, visual sightings, and maps. For safety, always resolve any discrepancies or questions before continuing navigation and defer to posted road signs.

Do not become distracted by the unit while driving, and always be fully aware of all driving conditions. Minimize the amount of time spent viewing the unit's screen while driving and use voice prompts when possible. Do not input destinations, change settings, or access any functions requiring prolonged use of the unit's controls while driving. Pull over in a safe and legal manner before attempting such operations.

The unit is designed to provide route suggestions. It is not designed to replace the need for driver attentiveness regarding road closures or road conditions, traffic conditions, weather conditions, or other factors that may affect safety of driving [2].

There are three safety issues being identified: be aware of all navigation sources, do not become distracted by the IVNS, and take IVNS directions as suggestions - not commands.

The first safety issue encourages comparing IVNS directions with other navigation sources. There is a warning to defer to posted signs when conflicts arise with IVNS. This warning is important because it establishes that conflicts do exist and that despite all the technology that goes into IVNS directions they can be wrong. 
The second safety issue, highlighted in bold, is a warning not to become distracted by the IVNS. One way to become distracted is to try and operate the device while driving. IVNS users are warned not to do this and to safely pull over to the side of the road if they need to operate the IVNS. The other form of distraction is looking at the IVNS screen while driving. The safety warning seems to contend that glances to the screen are inevitable as it instructs people to minimize, and not eliminate, this behaviour. The screen provides useful navigation information making it a tempting distraction. This information may aid a driver but if a glance turns into a stare then the results could be dangerous. Distraction is not a trivial matter as it is estimated to play a factor in anywhere from $25-80 \%$ of all crashes $[3,4]$.

The third safety issue concerns IVNS short comings regarding traffic, construction, weather, or any road conditions that can impact driving. This warning reiterates the need to pay attention to the road but also points out that an IVNS is not aware of everything and it is up to the driver to ultimately decide what is best. Instead of route directions they use the term route suggestions to make the point that is more important to follow the road than the IVNS.

It may seem intuitive that people should pay attention to their surroundings and that IVNS routes can be wrong; however, the media has reported several incidents that illustrate otherwise. An ambulance was supposed to make a 30 minute drive to a hospital 12 miles away in Brentwood, Essex. Instead, the IVNS routed the drivers to Brentwood, Manchester, and it was not until they had driven for four hours before they realized the mistake [5]. Another example involved a pair of car thieves who were apprehended when their IVNS routed them to a border crossing, and right into the hands of the law [6]. It is amazing that the car thieves did not notice that they were approaching the border until they were at the crossing. A twenty year old student, who was borrowing her boyfriend's car, ended up driving onto train tracks where the car was struck by a train [7]. "I put my complete trust in the sat nav," she explained, "and it led me right into the path of a speeding train." This occurred after she approached a gate with a sign that stated: "if the light is green, open the gates and drive through." She opened the gate and drove through. When she got out of her car to close the gate the train struck. Apparently until that point she was not aware that she was at a railroad crossing as it did not show up on the IVNS: "Obviously I had never done the journey before so I was using the sat nav - completely dependent on it." The driver did concede that the IVNS was not completely at fault, and that she had to take some responsibility for what happened:

I can't completely blame the sat nav because up until there, it did get me where I needed to go. If maybe I had been more aware of the situation, I wouldn't have had the accident. But I would be a bit more wary of the sat nav next time because they try to take you the shortest route, and not always the most accessible route and not always the safest route.

Perhaps the most astonishing thing about the above scenario is that a similar incident happened in the United States. A computer consultant who was driving a rental car made a right turn as advised by an IVNS. This led him onto train tracks where his car became stuck. He was able to get out of the car before a train crashed into it; pushing it more than 30 meters [8].

In all these cases the drivers should have been more aware of where they were going, but they blindly followed the IVNS. There is no indication that the typical IVNS user blindly follows their system, but it happened often enough in the village of St. Hillary, United Kingdom, that the local council erected a sign to warn drivers not to follow their IVNS [9]. A local traffic engineer explained the situation:

We've had a series of problems with drivers getting into trouble by trusting their satnav - and we needed to do something about it. I hope my sign should do the trick.

The problem he was referring to is that the IVNS was directing drivers down a narrow lane that is unsuitable for heavy trucks, with more than a dozen becoming stuck and causing traffic jams for hours.

However, signs are not always enough. The following story illustrates how an IVNS user completely ignored road signs while driving:

\section{A week ago, I saw a car drive the wrong way on a main street in Montreal.}

At the corner, another car coming from the correct direction flashed his lights to try to alert the errant motorist, who then started to turn at the intersection, again the wrong way on another one-way street. I waved my arms; he stopped and rolled down his window.

When I explained that both streets were one-way, he replied: "I can't go here? But my GPS system says to go on these streets." I told him, "You have to use your eyes!" and pointed to the one-way sign directly in front of us [10].

It seems that the man was amazed the IVNS could be wrong, that he should take IVNS information as merely route suggestions, not as absolute facts. It is unknown how long he had been using the IVNS, but many of the above incidents involved people who were new to the systems. The ambulance drivers were new to the job, the student who drove onto the train tracks was borrowing the car, and the computer consultant who drove onto the train tracks was in a rental car. Perhaps experienced IVNS users would have behaved differently.

The aim of our study is to understand if these incidents are abnormalities exaggerated in the media, or if IVNS users are blindly following their systems en masse. 


\section{METHOD}

A questionnaire was designed to provide insight into the measuring IVNS trust, the context for IVNS use, as well understand why IVNS may be used improperly, or not used at all. For the purposes of this paper we will focus on why participants used an IVNS.

Demographic questions were asked to understand who was answering the survey and account for difference of age, gender, and experience. Development of the questionnaire started by examining NaviQ an online questionnaire to study IVNS satisfaction [11]. We limited our survey to 44 questions to make it less time consuming for respondents. We asked questions to understand when people use, misuse, and disuse IVNS and have participants rate their trust in IVNSs.

Jian, Bisantz and Drury [12] created a questionnaire to measure trust. In order to create this questionnaire they conducted a word elicitation study where participants generated large set of words related to trust. These words were then rated by a separate group of participants for their relevance with trust or distrust. Lastly, 30 words that were highly rated for trust and distrust were used in a paired comparison study. A factor analysis was then conducted to create 12 clusters of words. We used these questions as the basis for our measurement of trust. In order to examine the specificity of IVNS trust additional trust ratings were collected about IVNS point of interest information.

The entire questionnaire was piloted with four IVNS users known to the investigators to ensure that the directions were clear and the questions were easy to understand. This process led to some questions being reworded and other questions being omitted altogether.

IMPLEMENTATION - Participants were primarily recruited from online communities of IVNS users, while some were also recruited from the community. Online communities were found by conducting a search at www.google.ca for "forum" and keywords such as "GPS", "car", and "navigation". Forum rules were read to determine if posting a request for survey participants was appropriate. When applicable, forum moderators were contacted to ask for permission. Participants accessed the survey by clicking on a link in the posting. The link directing them to the first page of the survey also acted as the consent form.

Participants were asked to give their consent, confirm that they were at least 18 years of age, and that they had used an IVNS. Participants who answered affirmatively were presented with the remainder of the survey. Participants of the online survey did not receive any compensation.

The questionnaire was also used in a series of interviews that allowed participants to provide more in-depth feedback than in an on-line survey. Interview participants were recruited from southern Ontario. Unlike the online survey where participants were self selected an effort was made to capture representatives from all age groups and genders. Interviews consisted of the same questionnaire as in the electronic survey, but participants were allowed to ask questions and provide additional comments at any time. Interviews took approximately 30 minutes to complete. Participants received $\$ 10$ in remuneration.

\section{RESULTS}

\section{DEMOGRAPHICS}

Gender - The respondents were over $90 \%$ male, with 190 males participating compared to only 20 females. The amount of male respondents is not surprising given that in a previous IVNS survey [11] where participants were solicited from online forums there were 97\% (131 out of 135) male respondents. Although gender disparity in overall Internet use has dissipated [13] males tend to use specialty websites more than females [14]. Another explanation for the gender imbalance could be that males tend to use navigation systems more than females. The 20 female respondents did not show any significant traits in their responses to differentiate themselves from the males; however, the sample size is too small to make any conclusions.

Age - Age distribution by gender is shown in Figure 1. The survey managed to capture respondents of each gender for all age ranges, although as mentioned in the previous section there were only 20 female respondents in total. Figure 2 compares the distribution with that of US Internet users [15] and licensed drivers [16]. The comparison with the U.S. Internet Users and Drivers is meant to illustrate the type of age distribution that might be considered normal when responses are obtained through an Internet survey involving drivers. The comparison shows that the survey follows the general bell-shape trend of Internet users, but with fewer respondents on the tail-end $18-24$ and 65 or older categories than the distribution of Internet users or licensed drivers would suggest. The lower turnout from younger people may be a result of there being fewer IVNS users in that age category due to the cost of an IVNS, the forums targeted may have less people in that age range, or it may reflect an aversion to taking the survey. There may have been few respondents aged 65 years or older because new technology is not designed for older adults [17] and they do not see the need to adopt new technology [18]. Recruiting older drivers for IVNS studies has been a challenge for other researchers [19]. 


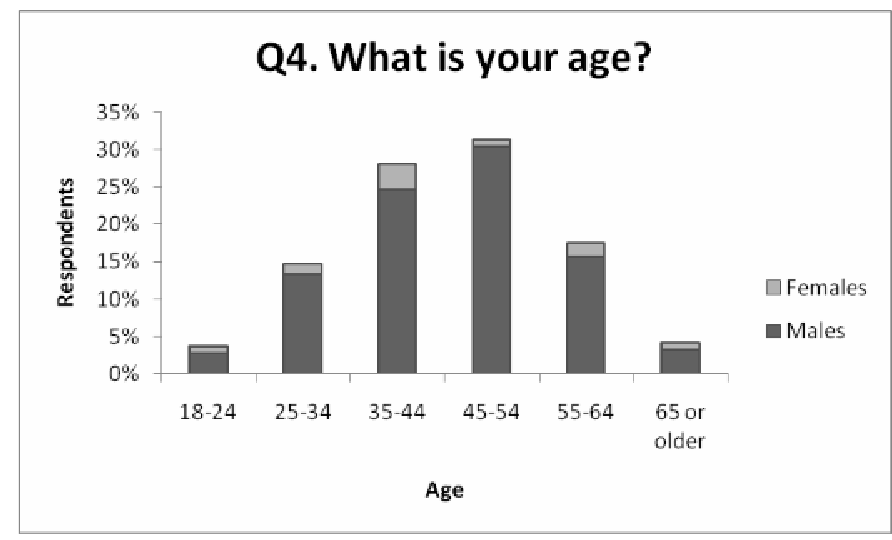

Figure 1 - The age distribution of respondents $(n=210)$.



Figure 2 - The age distribution of survey respondents compared with the age distribution for US home Internet users and licensed drivers.

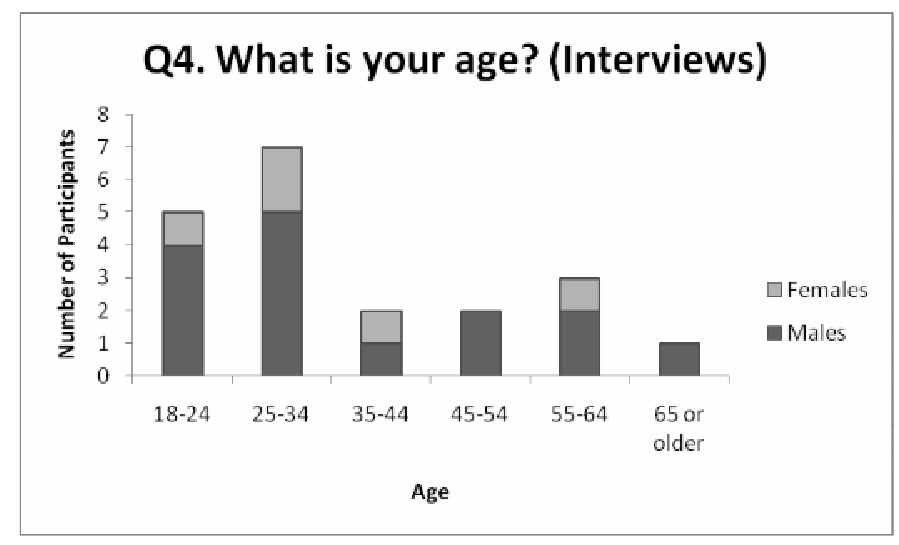

Figure 3 - Age and gender of interview participants.

The age and gender of interview participants is shown in Figure 3. There are representatives from each age range, but there are many more respondents in the 1824 and 25-34 categories, a reflection of the ease of recruiting participants from a university community compared to older groups.

Experience - In Q5 participants were asked "How often do you use an automotive navigation system?" and were given the choice of "Daily", "At least once a week", "At least once a month", and "Less than once a month". The results shown in Figure 4 are arranged from the most frequent users on the left side of the chart to the least frequent users on the right side of the chart. The majority of respondents are frequent users with over $60 \%$ using an IVNS once a week or more. There is also a good representation from both extremes with over $20 \%$ of respondents using an IVNS every day and almost $15 \%$ using an IVNS less than once a month. All but one driver drove at least once a week providing an opportunity to use an IVNS; however, not all of these drivers were in an unfamiliar area and $90 \%$ of respondents who reported driving in an unfamiliar area every day or every week use an IVNS at least once a week.

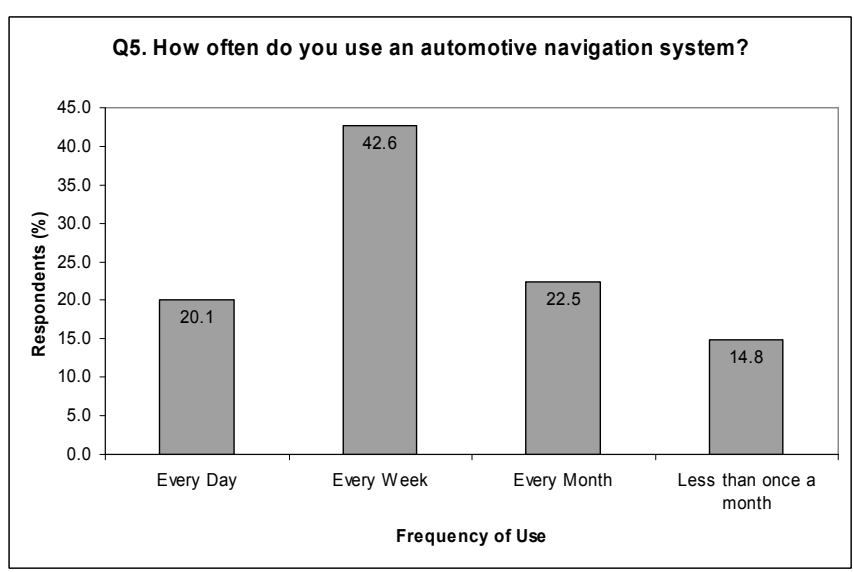

Figure 4 - Frequency of IVNS use $(n=209)$.

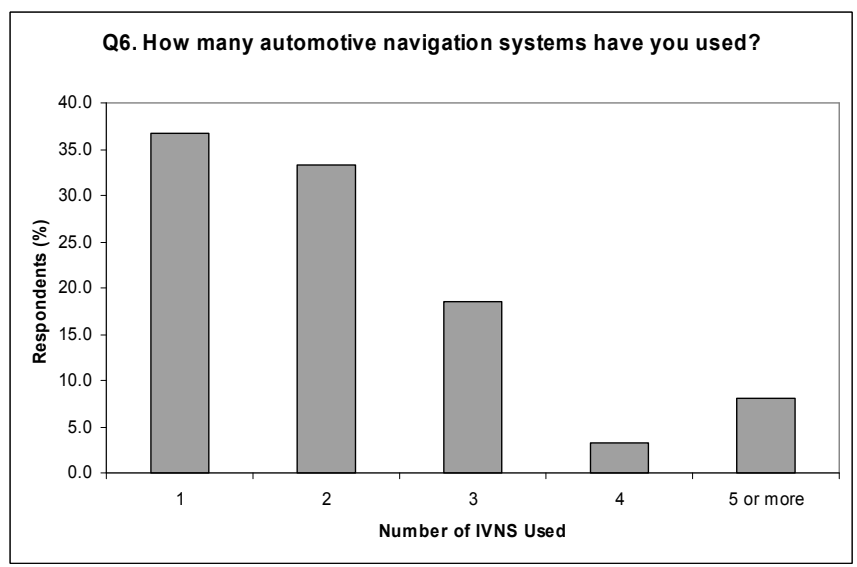

Figure 5 - Number of different IVNS Used $(n=210)$

It is important to know if respondents' views are based on interacting with only one IVNS or if they have encountered several systems. Experience with different systems gives people a better understanding of IVNS capabilities and should help them use the devices appropriately. The results of Q6 - "How many automotive navigation systems have you used?" shown in Figure 5 indicate that roughly a third have only used one IVNS, another third have used two systems and the remaining third have used 3 or more systems. Over $8 \%$ of respondents fall into a group of very experienced users who have used 5 or more IVNSs. Expert users are less likely to have problems with devices. 
Participants were asked how comfortable they are using an IVNS, computers, and driving, and for all three tasks at least $87 \%$ reported being comfortable, or very comfortable. The full results are shown in Figure 6 which also reveals that around $9 \%$ of participants are also very uncomfortable in all three areas. Using Kendall's tau-b test a substantial positive relationship was found between how comfortable participants are using an IVNS and how comfortable they are using a computer (tau- $b=$ .455 , Approx. Sig. $=.000, \mathrm{n}=209$ ). There is also a substantial positive relationship between how comfortable participants are using an IVNS and how comfortable they are driving (tau-b $=.393$, Approx. Sig. = $.000, \mathrm{~N}=209$ ). This means that the same people who are comfortable with IVNS are comfortable also comfortable driving and using a computer, while those who are uncomfortable using IVNS are uncomfortable driving or using a computer. Those who are uncomfortable are certainly in the minority, and that is not surprising given that the method used to recruit participants. Readers of online forums would be familiar with computers, and as previously stated they would tend to be enthusiasts who are more likely to be comfortable using a computer and with IVNS than the general population.

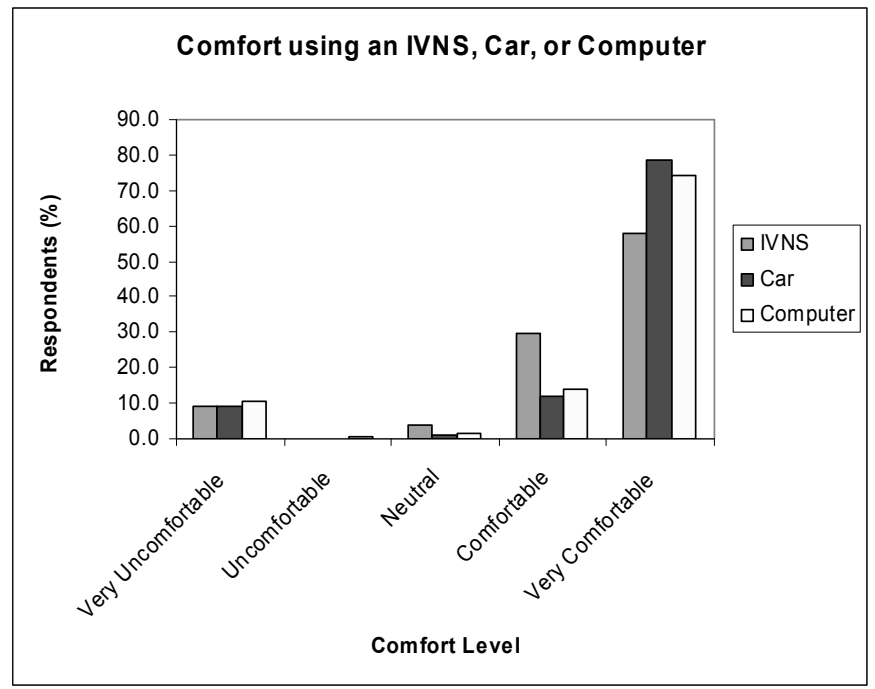

Figure 6 - A comparison of participants' comfort level using an IVNS, car, or computer $(n=210)$.

Participants rated their familiarity with IVNS on a 5 point Likert scale with 1 being not at all familiar, 5 being extremely familiar, and 3 being neutral. As shown in Figure $758.5 \%$ considered themselves to be extremely familiar with IVNS, while another $33.5 \%$ rated themselves as familiar. Kendall's tau-b test found a substantial positive relationship between comfort and familiarity with IVNS (tau-b $=.445$, Approx. Sig. $=.000, \mathrm{n}$ = 188). The fact that two-thirds of respondents have used more than one IVNS also demonstrates that the respondents can be characterized as experienced IVNS users. When examining the rest of the results it is important to keep in mind the results would probably be quite different if our respondents were novice IVNS users.

\section{TRUST}

The introduction gave several examples of situations where too much trust in the IVNS resulted in drivers ending up in precarious situations. We measured trust by asking both positive and negatively framed questions. As shown in Figure 7 around $90 \%$ of respondents rated the positively framed questions a 4 or 5 . The majority of responses for the negatively framed questions were either 1 or 2 as shown in Figure 8, but the percentage varied from as low as 71\% for Q33 - "I am suspicious of the navigation system's action or outputs" to $92 \%$ for Q35 - "The navigation system's actions will have a harmful or injurious outcome".

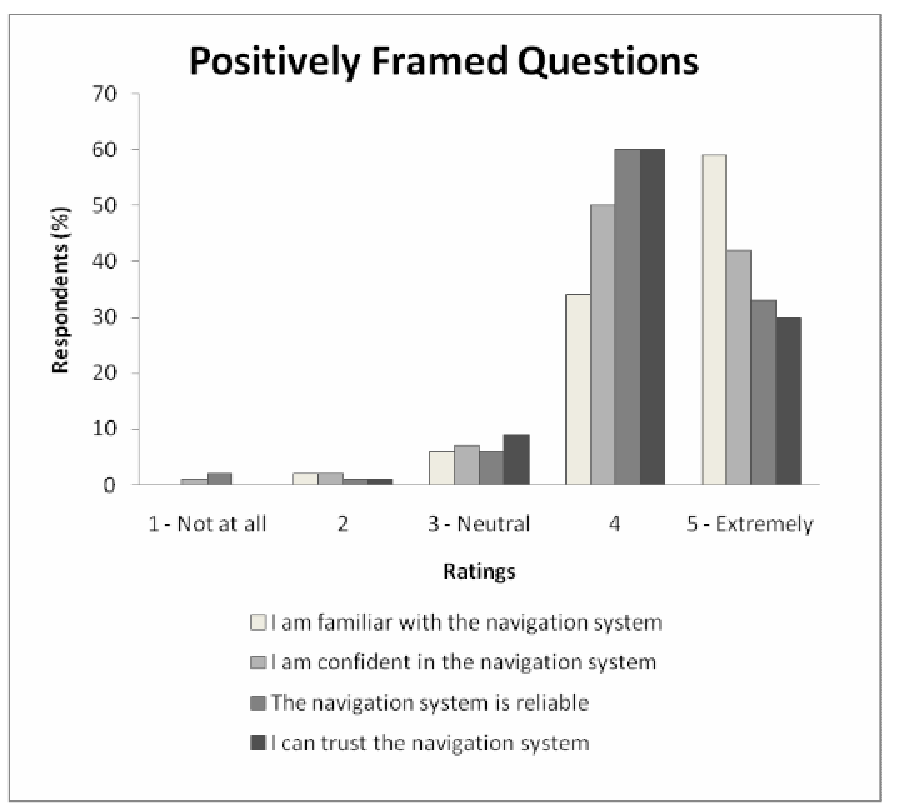

Figure 7 - Positively framed trust questions $(n=188)$.



Figure 8 - Negatively framed trust questions $(\mathrm{n}=188)$. 
Kendall's tau b test was performed on the responses and a very strong positive relationship was found between three of positively framed statements: "I am confident in the navigation system", "The navigation system is reliable", and "I can trust the navigation system" (Kendall's tau-b $>=.634$, sig. $=.000)$. Both the high ratings for the positively framed statements and the low ratings for the negatively framed statements indicate that the respondents have a high level of trust when using an IVNS.

\section{ATTITUDES TOWARDS SAFETY}

A high level of trust does not necessarily mean that too much faith is placed in IVNS directions. In order to address if the amount of trust that respondents have in IVNS is appropriate participants were asked a series of questions about what directions they followed when a conflicts arose between IVNS, people, and road signs. Figure 9 shows that $45.8 \%$ of respondents have followed an IVNS when it conflicted with advice from a person, but $41.5 \%$ have followed a person when it conflicted with advice from an IVNS. Road signs were more authoritative with $31.9 \%$ of respondents having followed an IVNS instead, and only $27.4 \%$ reported following directions from a person when it conflicted with a road sign.

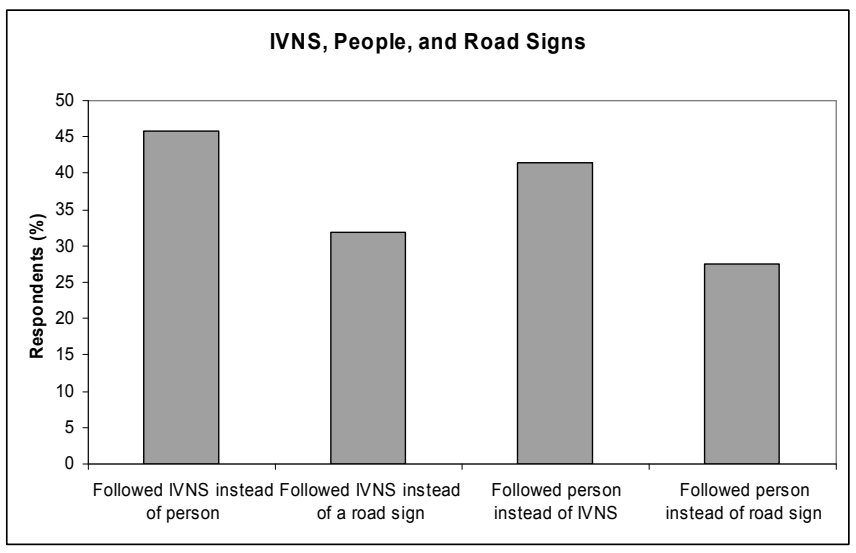

Figure 9 - Respondents were asked if they had followed an IVNS when it conflicted with a person or road signs, and also if they had followed directions from a person when it conflicted with IVNS or a road sign.

Participants also provided examples of when following all three sources turned out to be the right decision and times when they should have done the opposite. A common reason for following an IVNS instead of road signs is that the IVNS would provide a better route:

That was one of the situations that got me into trouble. The highway department had changed the exits so the maps in the unit were incorrect.

Other respondents made it clear that each incident is different and that it is always important to use common sense:
It does make mistakes - it's up to me to check the info I get from it.

One person mentioned serious mistakes that had occurred:

I have seen it tell me to turn when there was a jersey barrier preventing a turn, and a couple other times when turning isn't actually possible.

This participant's awareness that their IVNS might direct them to make an improper turn is good, but if another IVNS user takes an instruction as a command the results could be dire. Respondents provided some indications that they are less attentive to road signs when using their IVNS:

I rarely look at street signs unless the gps tells me to do something illegal. Illegal right turn or something.

While another respondent remarked:

When I am following the nav, I do not give priority to road signs.

Although incidences were mentioned when an IVNS gave illegal or incorrect instructions respondents also say that the device works correctly in most cases. Participants are willing to accept small errors occasionally made by the system:

Roads are always changing, so minor "mis-steps" by a navigation device are not outside reason.

Small errors may even be a good measure at preventing drivers from becoming too reliant on IVNS. Not a single respondent indicated they would blindly follow an IVNS, but some respondents are more inclined to follow the IVNS than other information sources.

Respondents felt there was little/no risk involved - There was very little concern about the possibility of IVNS use being unsafe with $78 \%$ of the respondents in our study thought that an IVNS is not at all harmful. In fact, only one respondent felt stronger than neutral about the possible danger of using an IVNS.

Experienced IVNS users feel that they are aware enough not to be led astray by the IVNS directions, and several stated that common sense must be used in all circumstances. This is a different attitude than the student described in the introduction who put her "complete trust" in the IVNS when using it for the first time. Respondents in our study know that although their devices are highly accurate they do make mistakes from time to time.

Only $8 \%$ of respondents felt that distraction was a problem when using an IVNS. It may be that experienced IVNS users know better than to manually operate the device while driving, pay too much attention to the display screen, or be distracted by the voice commands. 
Another possibility is that IVNS users are being distracted although they are not aware of it. A study on driver distraction due to cell-phones and perception of performance found that drivers underestimate the negative effects distraction has on their driving [21].

Some people drive differently with an IVNS - Some of our participants mentioned they pay less attention to road signs when using an IVNS. It would be interesting to know how widespread this tendency is among IVNS users. Paying less attention to signs can be seen as a benefit to safe driving or a detriment. The benefit is that drivers spend less time searching intersections for street signs so that they do not miss a turn, or scanning buildings for addresses. They can also spend less time thinking about logistics, debating what exit to take on the highway, As a result, drivers can spend more time with their eyes, and attention on the road. The downside is that they may miss signs that are important. Can people filter out the signs that are made redundant by the IVNS and still pay attention to the ones that matter?

It is also possible that some IVNS users are paying less attention to road signs and do not even realize it. This is one problem with self-reporting. If people spend less time looking at signs where they may spend more time looking at the road, the IVNS, their cell phone, or somewhere else entirely. These types of question lend themselves to eye tracker experiments, but even further interview questions on the subject may be fruitful.

Another concern is that IVNSs may make people forget how to navigate. One participant mentioned how a relative had become completely dependent on their IVNS. When it was taken in for repair they had trouble finding their way to places they had been driving to for decades. It is possible that by paying less attention to navigational cues with an IVNS, you forget to pay attention to navigational cues without it, or that without having to call upon routes by memory you the information no longer remains fresh and is lost.

\section{FUTURE WORK}

Our study elicited responses from experienced IVNS users from many different age groups. There were some groups who were under-represented, mainly females, 18-24 year olds and those over 65 years of age. A simple extension of our study would focus on any of these groups using different recruiting methods. It may also be beneficial to create a new questionnaire or add questions that specifically target an age group or address gender issues.

One of the respondents in our study reported that the safety of using an IVNS instead of stopping to ask directions was important to her because she was female. While male respondents also cited safety issues with strangers this may be more important to female IVNS users.
While not explicitly asked in our study, one respondent made a comment about the benefit of knowing when to turn ahead of time becomes more important as they get older:

As I am aging, I appreciate that my GPS helps me better anticipate maneuvers ("in .3 miles exit left").

There may be other aspects of IVNSs that are particularly appealing to older, younger, or female drivers that our study would have missed.

\section{CONCLUSION}

There is a discrepancy between drives' attitudes towards safety and potential dangers of using an IVNS. Drivers may be unaware of how distraction affects their driving. Some did not feel using an IVNS was dangerous at all because they are ultimately responsible for any incidents that may occur, and as one respondent stated: "It's up to me to check the info I get from it." The perceived risk of using an IVNS is low or almost non-existent as respondents are not concerned about distraction or that the IVNS will lead them astray. They understand IVNSs are not perfect and described errors they have encountered. Experienced IVNS users have a great deal of trust in their systems because they find the directions to be more reliable than other sources. Disregard for other navigation is a potentially dangerous habit as drivers could miss important cues. Overall drivers have a good common-sense approach to using an IVNS, but they may not be aware of how an IVNS may affect their driving.

\section{ACKNOWLEDGMENTS}

Funding for this research was provided by Auto 21 .

\section{REFERENCES}

1. A. Mccartt, L. Hellinga and K. Bratiman, "Cell Phones and Driving: Review of Research," Traffic Injury Prevention, vol. 7(2), pp. 89-106, 2006.

2. Garmin, "Important Safety and Product Information Sheet", Aug, 2007.

3. C. Nowakowski, P. Green and O. Tsimhoni, "Common Automotive Navigation System Usability Problems and a Standard Test Protocol to Identify Them," Transportation Research Institute, vol. 16, 2003.

4. V.L. Neale, T.A. Dingus, S.G. Klauer and J. Sudweeks, "An Overview of the 100-Car Naturalistic Study and Findings", Proc. Int. Tech. Conf. Enhanced Safety Vehicles, vol. 19, pp. 1-10, 2005.

5. Sun Online, "Satnav ambulance 200 miles out", Sun Online, 2 December, 2006, 27 July, 2007. http://www.thesun.co.uk/sol/homepage/news/article7 3749.ece

6. J. Wilkes, "GPS getaway a bum steer", thestar.com, 20 March, 2007, 21 March, 2007. http://www.thestar.com/printArticle/193790 
7. "Sat nav driver's car hit by train," BBC NEWS, 11 March, 2007, 11 March, 2007. http://news.bbc.co.uk/go/pr/fr/-

/2/hi/uk news/wales/south west/6646331.stm

8. Associated Press, "Man using GPS drives into path of train", MSNBC.com, 3 January, 2008. http://www.msnbc.msn.com/id/22493399/

9. Daily Mail, "Council erects sign that tells drivers: Don't trust your satnav", August 27, 2007. http://www.dailymail.co.uk/pages/live/articles/news/n ews.html?in article id=478073\&in page id =1770

10. S. Kazenal, "At the mercy of GPS," The Globe and Mail, Toronto, Ont.: Feb 4, 2008. pg. A.12

11. C. Li, NaviQ-A User Satisfaction Questionnaire for In-Vehicle Navigation Systems, Unpublished master's thesis, University of Guelph, 2006.

12. J.Y. Jian, A.M. Bisantz and C.G. Drury, "Foundations for an empirically determined scale of trust in automated systems", International Journal of Cognitive Ergonomics, vol. 4(1), pp. 53-71, 2000.

13. H. Ono and M. Zavodny, "Gender and the Internet", Social Science Quarterly, pp. 111-121, 2003.

14. R. Joiner, J. Gavin, J. Duffield, M. Brosnan, C. Crook, A. Durndell, P. Maras, J. Miller, A.J. Scott and P. Lovatt, "Gender, Internet identification, and Internet anxiety: correlates of Internet use," Cyberpsychology \& Behavior, vol. 8(4), pp. 371-378, 2005.

15. U.S. Census Bureau, "Computer and Internet use in the United States: 2003", U.S. Department of Commerce, October 2005. http://www.census.gov/prod/2005pubs/p23-208.pdf

16. National Safety Council, "Injury Facts, 2006”, 2006.

17. P. Gregor, A. Newell and M. Zajicek, "Designing for dynamic diversity: Interfaces for older people," Proceedings of the fifth international ACM conference on Assistive technologies, pp. 151-156, 2002.

18. N. Selwyn, S. Gorard, J. Furlong, and L. Madden, "Older adults' use of information and communications technology in everyday life," Ageing \& Society, vol. 23, pp. 561-582, 2003.

19. Green, P. "Variations in Task Performance Between Younger and Older Drivers: UMTRI Research on Telematics", Paper presented at the Association for the Advancement of Automotive Medicine Conference on Aging and Driving, February 19-20, 2001.

20. S. Katz, J. Fleming, P. Green, D. R. Hunter and D. Damouth, "On-the-Road Human Factors Evaluation of the Ali-Scout Navigation System (Technical Report UMTRI-96-32)," Ann Arbor, MI: The University of Michigan Transportation Research Institute, 1996.

21. W.J. Horrey, M.F. Lesch, A. Garabet and M.A. Hopkinton, "Awareness of Performance Decrements Due to Distraction in Younger and Older Drivers", Proceedings of the Fourth International Driving Symposium on Human Factors in Driver
Assessment, Training and Vehicle Design, Stevenson, Washington, pp. 54-60, 2007.

\section{CONTACT}

Andrew Varden may be contacted by email: avarden@gmail.com. 